\title{
Über den Einfluss der Avertinnarkose auf die Phagozytose der Leukozyten.
}

\author{
Von \\ Tsunehiko Aoki. \\ (青木霜彦) \\ (Aus der Chirurgischen Klinik der Kaiserlichen Universität \\ zu Sendai. Dircktor: Prof. Dr. Sh. Sekiguchi.)
}

\section{Einleitung.}

Die folgende Abhandlung ist ein Bericht über experimentelle Untersuchungen an Meerschweinchen mit Avertin, dem vorzüglichen, neuen, rektalen Narkotikum. Die Versuche wurden mit der Absicht angestellt zu untersuchen, welchen Einfluss das Avertin im Vergleich zu den bisher vorhandenen Narkotika auf die Phagozytose der Leukozyten hat, da es in der klinischen Praxis sehr wichtig sein dürfte, die Verhältnisse bei einer postnarkotischen Infektion zu kennen und den Heilvorgang beurteilen zu können.

\section{Vorversuch : U̇ber die Dosierung der Avertinnarkose beim Meerschweinchen.}

Zunächst wurde die geeignetste Dose für die rektale und intravenöse Avertinnarkose beim Meerschweinchen bestimmt.

Die verwendete Avertinlösung ist die, welche liquides Avertin frisch dem Aqua destillata zugesetzt, dieses bei $40^{\circ} \mathrm{C}$ in einer Menge von $2,5 \%$ gelöst enthält.

Beim Versuch der rektalen Narkose führt man die beinahe bis zur Körpertemperatur erwärmte Avertinlösung mit dem Nélatonschen Katheter in den Rektum hinein und drückt den Analteil einige Minuten lang nach der Einführung zu, um den Ausfluss der Lösung zu verhindern. Beim Versuch mit intravenöser Narkose injiziert man 
die Avertinlösung in die blossgelegte, rechte Jugularvene und näht die Haut sofort nach der. Injektion zu.

\section{Tabelle 1.}

Versuchsresultat der rektalen Avertinuarkose.

\begin{tabular}{|c|c|c|c|c|c|c|c|}
\hline 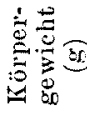 & $\mid \begin{array}{ll}1 & \\
0 & 0 \\
0 & 0 \\
0 & 0 \\
0 & 0\end{array}$ & $\begin{array}{l}\text { Avertin- } \\
\text { dose pro } \\
\mathrm{kg}(\mathrm{g})\end{array}$ & Narkosendauer & 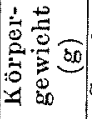 & 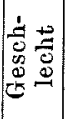 & $\begin{array}{c}\text { Avertin- } \\
\text { dose pro } \\
\mathrm{kg}(\mathrm{g})\end{array}$ & Narkosendauer \\
\hline 460 & $q$ & 0,01 & (Nicht geschlafen) & 475 & 우 & 0,25 & $1 \mathrm{St} .30 \mathrm{Min}$. \\
\hline 380 & $\delta$ & 0,02 & (Nicht geschlafen) & 380 & 今 & 0,25 & $2 \mathrm{St}$. \\
\hline 410 & 9 & 0,05 & (Nicht geschlafen) & 540 & 9 & 0,25 & 1 St. $10 \mathrm{Min}$. \\
\hline 430 & $\hat{b}$ & 0,05 & 3 Min. & 380 & $\delta$ & 0,3 & B St. \\
\hline 510 & 3 & 0,05 & (Nicht geschlafen) & 420 & 웅 & 0,3 & 2 St. $20 \mathrm{Min}$. \\
\hline 382 & $\hat{0}$ & 0,1 & $15 \mathrm{Min}$. & 472 & 8 & 0,3 & $1 \mathrm{St} .55 \mathrm{Min}$. \\
\hline 480 & P & 0,1 & 7 Min. & 350 & o & 0,3 & $2 \mathrm{St} .30 \mathrm{Min}$. \\
\hline 417 & 8 & 0,1 & $10 \mathrm{Min}$. & 418 & $\delta$ & 0,35 & (Gestorben) \\
\hline 420 & $\hat{0}$ & 0,15 & $35 \mathrm{Min}$. & 500 & 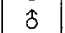 & 0,35 & 2 St. 40 Min. \\
\hline 490 & 8 & 0,15 & 50 Min. & & & 035 & (f St. 20 Min. \\
\hline 394 & s & 0,15 & $46 \mathrm{Min}$. & 518 & 우 & 0,35 & (Künstliche Atmung) \\
\hline 506 & + & 0,15 & 1 St. & 460 & 우 & 0,4 & (Gestorben) \\
\hline 400 & 웅 & 0,2 & $1 \mathrm{St} .15 \mathrm{Min}$. & & & & $\int \quad 3 \mathrm{St}$ \\
\hline 550 & $q$ & 0,2 & $50 \mathrm{Min}$. & 440 & $\delta$ & 0,4 & ('(Künstliche Atmung) \\
\hline 426 & $\hat{\jmath}$ & 0,2 & 1 St. $20 \mathrm{Min}$. & 390 & 온 & 0,5 & (Gestorben) \\
\hline
\end{tabular}

Beim Versuch mit rektaler Narkose (Tab. 1) wird das Meerschweinchen durch 0,01-0,05 g Avertin pro kg Körpergewicht fast nicht und durch $0,1 \mathrm{~g}$ leicht betäubt; mit $0,15-0,3 \mathrm{~g}$ wird eine geeignete Narkose erzielt; eine Dose von $0,35 \mathrm{~g}$ und darüber überlebt es selten, und manchmal stirbt es bald durch Herzlähmung, auch wenn künstliche Atmung angewendet wird.

Tabelle 2.

Versuchsresultat der intravenösen Avertinnarkose.

\begin{tabular}{|c|c|c|c|c|c|c|c|}
\hline 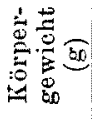 & 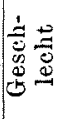 & $\begin{array}{c}\text { Avertin- } \\
\text { dose pro } \\
\text { kg (g) }\end{array}$ & Narkosendauer & 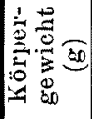 & $\begin{array}{ll}\frac{1}{0} & = \\
0 & 0 \\
0 & 0 \\
0 & 0\end{array}$ & $\begin{array}{c}\text { Avertin- } \\
\text { dose pro } \\
\mathbf{k g}(\mathbf{g})\end{array}$ & Narkosendauer \\
\hline 465 & $\delta$ & 0,02 & (Nicht geschlafen) & 500 & 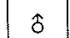 & 0,08 & $55 \mathrm{Min.}$ \\
\hline 420 & $\hat{\delta}$ & 0,02 & (Nicht geschlafen) & 398 & 우 & 0,1 & $1 \mathrm{St}, 30 \mathrm{Min}$. \\
\hline 384 & \% & 0,05 & 18 Min. & 460 & $\%$ & 0,1 & $2 \mathrm{st} .5 \mathrm{Min}$. \\
\hline 475 & q & 0,05 & $12 \mathrm{Min}$. & 415 & 우 & 0,1 & 1 St. 10 Min. \\
\hline 402 & 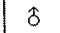 & 0,05 & 20 Min. & 444 & & 0.15 & $\int 3 \mathrm{St}$ \\
\hline 500 & 유 & 0,08 & 1 St. & 444 & 0 & 0 , & (Künstliche Atmung) \\
\hline 496 & q & 0,08 & 1 St. 10 Min. & 510 & $\delta$ & 0,15 & (Gestorben) \\
\hline 390 & $\delta$ & 0,08 & 40 Min. & 430 & $\delta$ & 0,2 & (Gestorben) \\
\hline
\end{tabular}


Beim Versuch mit intravenöser Narkose (Tab. 2) wird das Meerschweinchen durch $0,02 \mathrm{~g}$ Avertin pro kg Körpergewicht nicht und durch $0,05 \mathrm{~g}$ leicht betäubt; mit $0,08-0,1 \mathrm{~g}$ wird eine geeignete Narkose erzielt; eine Dose von $0,15 \mathrm{~g}$ und darüber überlebt es nicht auch bei Anwendung künstlicher Atmung.

Aus den zwei obigen Versuchsresultaten ist ersichtlich, dass die beste Dose für die rektale und intravenöse Avertinnarkose beim Meerschweinchen $0,15-0,3 \mathrm{~g}$, bzw. 0,08-0,1 g pro kg Körpergewicht ist. Vergleicht man diese Mengen mit den von Eichholtz ${ }^{1}$ für verschiedene Tierarten bestimmten Avertindosen, so erscheint es wahrscheinlich, dass das Meerschweinchen gegen diese Narkotika etwas empfindlicher ist als das Kaninchen, der Hund und die Maus.

\section{Kombinierter Verstech in vivo et in vitro.}

Im diesen Abschnitte wurde die Veränderung der Phagozytose dadurch beobachtet, dass die Leukozyten und das Serum eines Meerschweinchen, an dem die rektale Avertinnarkose ausgeführt war, mit der Bakterienflüssigkeit im Reagenzglas in Berührung gebracht wurden.

\section{Versuchsmaterial und -method.}

Versuchsmaterial: Als Versuchstiere wurden gesunde, männliche Meerschweinchen von ca. $400 \mathrm{~g}$ Körpergewicht verwendet. Zur Herstellung der Bakterienemulsion wurde der von einem Furunkel, einer Myositis, einer Mastitis etc. erhaltene Staphylococcus aureus angewandet. Und zwar wurde eine Platinöse voll des auf $37^{\circ} \mathrm{C} 24$ Stunden lang am schiefen Nähragar rein kultivierten frischen Bakterienstamms mit $5 \mathrm{ccm}$ sterilisierter $0,85 \%$ iger Kochsalzlösung abgespült, und genügend stark und lange geschüttelt. Nach einer folgenden 30 Minuten langen Erwärmung und Sterilisation in einem Wasserbad von $60^{\circ} \mathrm{C}$, wurde die Bakterienemnlsion geeignet verdünnt, dass sie in $1 \mathrm{ccm}$ etwa $10^{9}$ Bakterien enthält. Die verwendete Avertinlösung ist dieselbe wie die beim Vorversuch. Etwa 1,5 ccm Blut wird jedesmal vom Herzen des Meerschweinchens entnommen. Die Leukozytenaufschwemmung wird dadurch erhalten, dass immer genau 5 Tropfen des Blutes aus einer Spritze in ein vorher sterilisiertes, getrocknetes Zentrifugierspitzglas mit $1 \mathrm{ccm}$ 1,5\%iger Natriumzitratlösung getropft und vorsichtig gemischt werden. Das Serum wird vom übrigen Blut (ca. $1 \mathrm{ccm}$ ) gewonnen.

Versuchsmethode: Zu einer bestimmten Stunde nach der Avertinnarkose entnimmt man das Blut mittels der Herzpunktion und nach der oben

1) Eichholtz, Deutsch. med. Wschr., 1927, 710. 
genannten Methode erhält man die Leukozytenauf schwemmung und das Serum.

Man führt erstens je $0,2 \mathrm{ccm}$ der Leukozytenaufschwemmung, der Bakterienemulsion und des Serums der Reihe nach in ein Reagenzgläschen ein und mischt genügend. Dann überlässt man es ca. 30 Minuten lang im Brütofen einer Temperatur von $37^{\circ} \mathrm{C}$. Wenn man endlich das Ausstrichpräparat aus diesem Röhrchen herstellt, fixiert man es in Leishmanns Lösung und färbt es. Beim Mikroskopieren bestimmt man die Prozentzahl der verschiedenen Leukozyten und bezeichnet die Zahl der jetzt bakterienfressenden Leukozyten als "p" unter 200 der im willkürlichen Gesichtsfeld gezähiten; die Zahl der in diesen aufgenommenen Bakterien wird als " $\mathrm{b}^{\prime \prime}$ bezeichnet, die Summe von , $\mathrm{p}^{\text {" und , }}$ " als , $\mathrm{f}$ " und die durchschnittliche Zahl der in einem Leukozyten aufgenommenen Bakterien als "s". (p) und (b) sind die echte Zahl, welche die Gesamtzahl zeigt. Da dje Lymphozyten und die Mastzellen im allgemeinen nicht phagozytiert sind, so wird hier nur die Prozentzahl der beiden angegeben.

Wenn die Avertindose beim Versuch zu gross ist, sterben die Tiere zuweilen unerwartet während des Versuchs.

Ich nahm 0,15 g Avertin pro kg Körpergewicht des Meerschweinchens als die mittlere Menge für Narkose an gemäss der im Vorversuch der rektalen Narkose erhaltenen Versuchsresultate und unterschied gemäss der Avertinmenge die nachfolgenden drei Fälle:

(1) Grosse Avertinmenge; $0,30 \mathrm{~g}$ pro $\mathrm{kg}$ Körpergewicht,

(2) Mittlere Avertinmenge; $0,15 \mathrm{~g}$ pro kg Körpergewicht,

(3) Kleine Avertinmenge; $0,05 \mathrm{~g}$ pro kg. Körpergewicht.

Als Kontrolle wurde die obige Methode an Meerschweinchen auch ohne Narkose angewandt.

\section{Versuchsresultate.}

Bei der Kontrolle (ohne Narkose-Tab. 6) ist die Phagozytose der Leukozyten leichtgradig gesteigert und die polymorphkernigen, neutrophilen Leukozyten vermehren sich auch leicht nach der Blutentnahme. Und zwar beginnen (p), (b), (f) und (s) in ihrem Werte nach 30 Minuten zu zunehmen, innerhalb 1-3 Stunden erreichen sie ihren maximalen Wert, nehmen aber dann allmählich ab und kehren nach 12 Stunden innerhalb Normbereiches zurück.

Bei mittlerer Avertinmenge (beste Dose der Narkose) zeigt der Einfluss auf die Phagozytose einen grossen Unterschied je nach der Individualität des Tieres. Eines zeigt anfänglich eine vorübergehende Hernmung der Phagozytose, später dagegen eine Steigerung, welche dann auf den Normalwert zurückgeht; ein anderes zeigt direkt 
Tabelle 3.

Versuchsresultat bei mittlerer Avertinmenge. (Durchschnittswert an 10 Tieren)

\begin{tabular}{c} 
Zeitverlauf \\
\hline
\end{tabular}

Tabelle 4.

Versuchsresultat bei grosser Avertinmenge.

(Durehschnittswert an 5 Tieren)

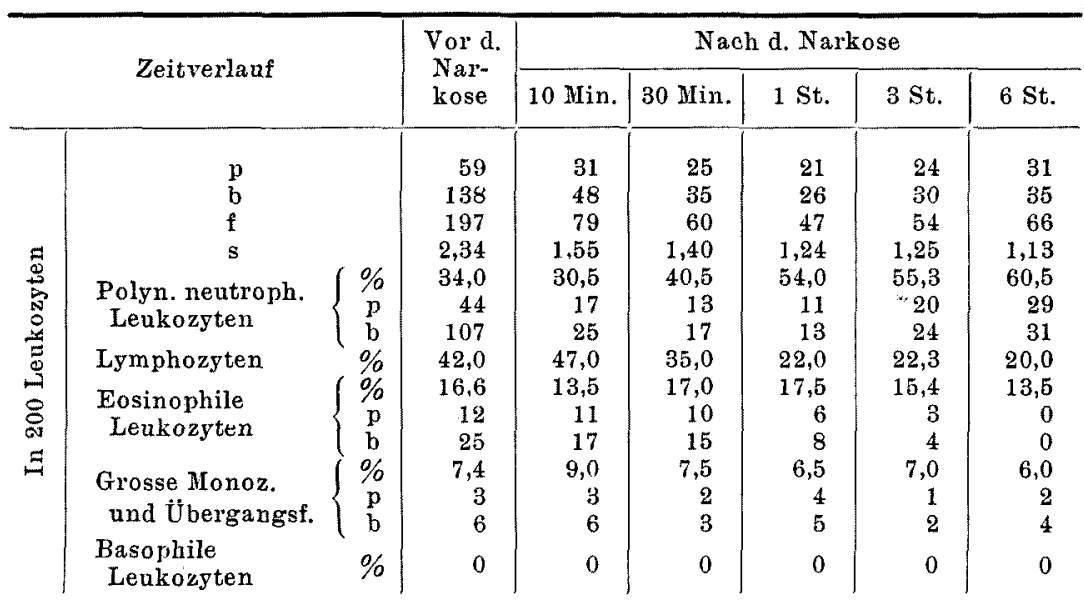

nach der Narkose keine Hemmung, sondern eine Förderung. Die durchschnittliche Beobachtung an 10 Tieren (Tab. 3) ergibt folgendes : (p), (b), (f) und (s) nehmen anfangs innerhalb kurzer Zeit (10 Min., 
Tabelle 5.

Versuchsresultat bei kleiner Avertinmenge.

(Durchschnittswert an 5 Tieren)

\begin{tabular}{|c|c|c|c|c|c|c|c|c|c|c|c|}
\hline & \multirow{2}{*}{\multicolumn{2}{|c|}{ Zeitverlauf }} & \multirow{2}{*}{$\begin{array}{l}\text { Vord. } \\
\text { Nal- } \\
\text { kose }\end{array}$} & \multicolumn{8}{|c|}{ Nach d. Narkose } \\
\hline & & & & 10Min. & 30 Min. & 1 St. & $3 \mathrm{St}$ & 6 St. & $12 \mathrm{St}$. & $24 \mathrm{St}$. & $48 \mathrm{St}$. \\
\hline \multirow{15}{*}{ 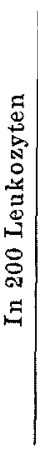 } & $\mathrm{p}$ & & 47 & 54 & 60 & 68 & 61 & 52 & 47 & 43 & 44 \\
\hline & $\mathrm{b}$ & & 88 & 111 & 126 & 150 & 132 & 110 & 93 & 81 & 80 \\
\hline & $f$ & & 135 & 165 & 186 & 218 & 193 & 162 & 140 & 124 & 124 \\
\hline & $\mathbf{s}$ & & 1,87 & 2,06 & 2,10 & 2,21 & 2,16 & 2,12 & 1,98 & 1,88 & 1,82 \\
\hline & Polynukleäre & $\%$ & 39,5 & 42,7 & 48,0 & 58,5 & 73,2 & 80,5 & 54,5 & 53,3 & 44,0 \\
\hline & neutrophile & $\mathrm{p}$ & 37 & 48 & 54 & 58 & 54 & 47 & 35 & 36 & 40 \\
\hline & Leukozyten & $\mathrm{b}$ & 70 & 99 & 116 & 132 & 116 & 97 & 72 & 69 & 75 \\
\hline & Lymphozyten & & 43,6 & 49,0 & 41,8 & 29,7 & 18,0 & 15,0 & 32,8 & 36,5 & 46,8 \\
\hline & Eosinophilo & $\%$ & 11,2 & 4,5 & 6,0 & 6,5 & 3,3 & 2,0 & 8,7 & 7,5 & 3,5 \\
\hline & Leukozyten & $\mathrm{p}$ & 9 & 5 & 5 & 9 & 4 & 3 & 8 & 6 & 2 \\
\hline & & $\mathrm{b}$ & 16 & 10 & 8 & 17 & 9 & 8 & 15 & 11 & 3 \\
\hline & Grosse Monoz. & $\%$ & 5,7 & 3,8 & 4,2 & 5,3 & 5,5 & 2,5 & 3,8 & 2,7 & 5,7 \\
\hline & und Uber'- & $p$ & 1 & 1 & 1 & 1 & 3 & 2 & 4 & 1 & 2 \\
\hline & gangsform & & 2 & 2 & 2 & 1 & 7 & 5 & 6 & 1 & 2 \\
\hline & Leukozyten & $\%$ & 0 & 0 & 0 & 0 & 0 & 0 & 0,2 & 0 & 0 \\
\hline
\end{tabular}

Tabelle 6.

Versuchsresultat bei Kontrolle-ohne Narkose.

(Durchschnittswert an 5 Tieren)

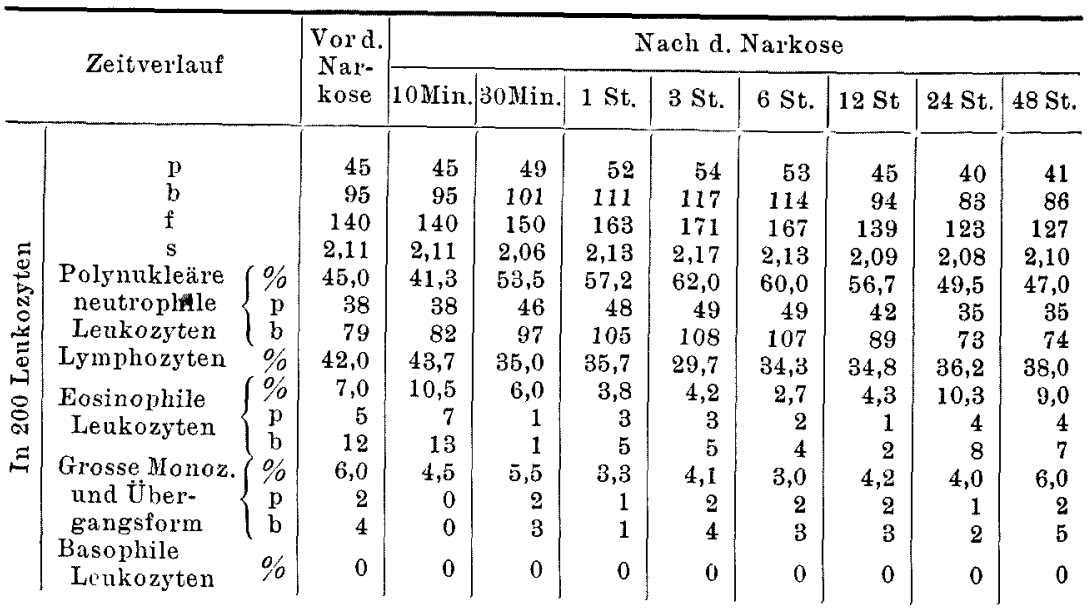

$30 \mathrm{Min}$.) alle in ihrem Werte $\mathrm{zu}$, sinken aber bis zu einem minimalen Wert nach 1 Stunde stark ab. Dann vermehren sie sich allmählich bis zum Normalwert innerhalb 6-12 Stunden. Nach weiteren 24 Stun- 
den zeigen sie eine Vermehrung über den Normalwert, der aber nach 48 Stunden wieder erreicht wird. Wenn auch die Phagozytose bei dieser Avertinmenge vorübergehend niedriger als beim Kontrollversuch zu sein scheint, so wäre es oberfächlich, dies als Regel anzusehen, weil die individuellen Unterschiede sehr auffallend wie oben angegeben sind.

Bei grosser Avertinmenge starb das Meerschweinchen längstens innerhalb 3-6 Stunden nach der Narkose an Atem- und Herzstillstand durch den merklichen Einfluss der Narkose selbst und grossen Eingriff, welchen die Herzpunktion für den Organismus darstellt. Daher wurde nur bis zu 6 Stunden nach Beginn der Narkose beobachtet. Bei grosser und kleiner Avertinmenge zeigt die Phagozytose so leichtgradige individuelle Unterschiede, dass sie im allgemeinen ähnlich verläuft, und im Vergleich mit der bei mittlerer Menge ohne merkliche Unterschiede. Und zwar vermindert sich bei grosser Menge (Tab. 4) das phagozytäre Vermögen deutlicher als beim Kontrollversuch sofort mach der Narkose, wogegen es sich bei kleiner Menge (Tab. 5) zu diesem Zeitpunkte vermehrt.

Es wäre wünschenswert, bei der Untersuchung des weissen Blutbildes zugleich mit dem Prozentsatz die absolute Zahl zu zählen. Da bei meiner Versuchsmethode die Gesamtzahl der Leukozyten unklar ist, so ist auch die absolute Zahl der verschiedenen Leukozytenarten unklar. Die Schwankungen des Prozentsatzes der Eosinophilen, der grossen Monozyten, sowie der U̇bergangsformen und der Mastzellen bei der Narkose sind bestimmt. Aber der Prozentsatz der Neutrophilen (Tab. 3, 4,5) vermehrt sich im allgemeinen stark innerhalb kurzer Zeit nach der Narkose unabhängig von der Avertindose sowie der Narkosenzeit und erreicht nach 6 Stunden den maximalen Wert. Der Grad dieser Vermehrung ist je nach der Avertinmenge verschieden und beträgt bei mittlerer, grosser und kleiner Menge bzw. $23,4 \%, 26,5 \%$ und $41,0 \%$; diese Werte sind alle grösser als beim Kontrollversuch mit 17,0\% (Tab. 6). Hierauf vermindert er sich allmählich und erreicht nach 48 Stunden den Normalwert. Beim Kontrollversuch sind die Schwankungen langsamer als bei Anwendung der Narkose und der maximale Wert wird nach 3 Stunden erreicht. Der Prozentsatz der Lymphozyten (Tab. 3,4,5) zeigt gerade die entgegengesetzten Schwankungen im Vergleich mit dem der Neutrophilen. Er zeigt jedoch im allgemeinen direkt nach der Narkose eine vorübergehende Vermehrung, dann eine sofortige rasche Verminderung und nach 6 Stunden das Erreichen des minimalen Wertes. Der Grad dieser Verminde- 
rung ist je nach der Avertinmenge verschieden und beträgt bei mittlerer, grosser und kleiner Menge bzw. 20,0\%,22,0\% und 28,6\%; diese Werte sind alle grösser als beim Kontrollversuch mit 12,3\% (Tab. 6). Hierauf wird der Prozentsatz der Lymphozyten allmählich grösser und erreicht nach 48 Stunden den Normbereich. Beim Kontrollversuch gewinnt er nach 3 Stunden, wie die Neutrophilen, den minimalen Wert.

\section{Versuch in vitro (1).}

In diesem Abschnitt wurden die Wirkungen von Avertinlösungen verschiedener Konzentration auf die Bakterienemulsion, die Leukozyten und das Serum eines nicht behandelten Meerschweinchens durch die direkte Berührung im Reagenzglas untersucht, um den Einfluss der Avertin-Konzentration auf eine Hemmung oder Förderung der Phagozytose festzustellen.

Versuchsmaterial und -methode.

Versuchsmaterial: Die Versuchstiere und die Bakterienemulsion sind dieselben wie im vorigen Abschnitte. Die Leukozytenaufschwemmung wird wie folgt hergestellt: Auf Körpertemperatur erwärmte Bouillon-Kochsalzlösung (1:1) wird in einer Menge von 0,75 ccm pro kg Körpergewicht einem Meerschweinchen intraperitoneal injiziert. Nach ca. 6 Stunden wird das intraperitoneale Exsudat mit der Kapillarpipette aufgesogen und in einer beinahe gleichen Menge 1,5\%iger Natriumzitratlösung aufgenommen, um einem Gerinnen vorzubeugen; dann wird sofort in einer Zentrifuge möglichst langsam zentrifugiert. Weiter, nach nur einmaliger spülung mit 0,85\%iger Kochsalzlösung und folgender Zentrifugierung stellt man die Konzentration des erhaltenen Sedimentes makroskopisch gleich der einer 0,3\%igen Lecithinlösung her. Das Serum wird in der Weise frisch gewonnen, dass man ca. $5 \mathrm{ccm}$ des durch Herzpunktion desselben Meerschweinchens erhaltenen Blutes defibriniert und zentrifugiert. Die verwendete Avertinlosung ist dieselbe wie beim Vorversuch. Ihre für diese Versuche zweckmässigste Konzentration ist die im Blute bei der Narkose vorhandene. Sebenin $\mathrm{g}^{2}$ ) allein berichtete über solch eine Konzentration, aber beschrieb nicht eingehend die Abhängigkeit der klinischen Befunde in jeden Stadium der Narkose zur Avertin-Konzentration im Blute.

Ich nehme daher bei diesen Versuchen die Avertindose der intravenösen Narkose als Grundlage. Als geeignetste Avertindose für die intravenöse Narkose beim Meerschweinchen betrügt nach den Resultaten des Vorversuches 0,08$0,1 \mathrm{~g}$ pro kg Körpergewicht.

2) Sebening, Schmerz, Narkose-Anästhesie, 1929-30, 2, 403. 
Wenn man das Verhältnis des Vollblutes zum Körpergewicht des Meerschweinchens nach der Schilderung in Wintersteins ${ }^{3)}$ "Handbuch der vergleichenden Physiologie" mit 1:18 annimmt und auf das Vollbut eines etwa $400 \mathrm{~g}$ wiegenden Tieres umrechnet, so ist es wahrscheinlich, dass man dieses Tier mit einer ca. 0,15-0,18 g\% igen Avertinmenge narkotisieren kann. Nehme ich nun die $0,15 \mathrm{~g} \%$ ige Avertinmenge, so beträgt ihre Konzentration in der Mischung (je $0,2 \mathrm{ccm}$ ) von Avertinlösung, Lueukozytenaufschwemmung, Bakterienemulsion und Serum nur $0,0375 \%$, welche nur $\$$ der vorigen entspricht. Also um die Avertin-Konzentration im Reagenzglas auf $0,15 \% \mathrm{zu}$ bringen, muss die Avertinlösung für den Versuch $0,6 \%$ ig sein.

Daher stellte ich hier eine 0,6\%ige Avertinlösung und die 7 nachfolgenden stärkeren oder schwächeren Lösungen her:

$\begin{array}{ccc}\begin{array}{c}\text { Nr. des } \\ \text { Reagenzglases }\end{array} & \begin{array}{c}\text { Konzentration der } \\ \text { Avertinlösnng fiir } \\ \text { den Versuch }(\%)\end{array} & \begin{array}{c}\text { Avertin-Konzen- } \\ \text { tration im } \\ \text { Reagenzlas }(\%)\end{array} \\ \text { I } & 0,1 & 0,025 \\ \text { II } & 0,3 & 0,075 \\ \text { III } & 0,4 & 0,100 \\ \text { IV } & 0,5 & 0,125 \\ \text { V } & 0,6 & 0,150 \\ \text { VI } & 0,7 & 0,175 \\ \text { VII } & 0,8 & 0,200\end{array}$

Versuchsmethode: Man bringt in 7 kleine Reagenzglaschen $0,2 \mathrm{ccm}$ von jeder Avertiulösung mit den oben genannten Konzentrationen ein und in ein weiteres Reagenzglas ebenfalls $0,2 \mathrm{ccm}$ physiologischer Kochsalzlösung als Kontrolle. In jedes Gläschen wird dann je $0,2 \mathrm{ccm}$ der Leukozytenaufschwemmung, der Bakterienemulsion und des Serums hinzugefügt und genügend ge. mischt. Nachdem man diese Mischung in den Brütofen hineinlegt hat, ist die Behandlung dieselbe wie im vorigen Abschnitte.

Versuchsresultate.

Nicht nur bei der durchschnittlichen (Tab. 7), sondern auch bei der einzelnen Beobachtung für alle in der Tabelle angeführten Avertin-Konzentrationen sind die Werte für (p), (b), (f) und (s) grösser als für den Kontrollversuch, d. h. die Phagozytose der Leukozyten wird fördernd beeinflusst. Die konzentrierteste Avertinlösung von $0,8 \%$ entspricht $0,11 \mathrm{~g}$ pro $\mathrm{kg}$ Körpergewicht des Meerschweinchens bei intravenöser Narkose. Diese Konzentration bewirkt klinisch eine ruhige Narkose, zuweilen Zyanose oder Atembeschwerden.

Im Prozentsatz der Leukozyten wurden keine grossen Unterschiede zwischen den einzelnen Fällen und dem Kontrollversuch be-

3) Wintersteins Handbuch der vergleichenden Physiologie, I-1, Jena 1925, 111. 
Tabelle 7 .

Versuchsresultat in vitro (1).

(Durchschnittswert an 5 Tieren)

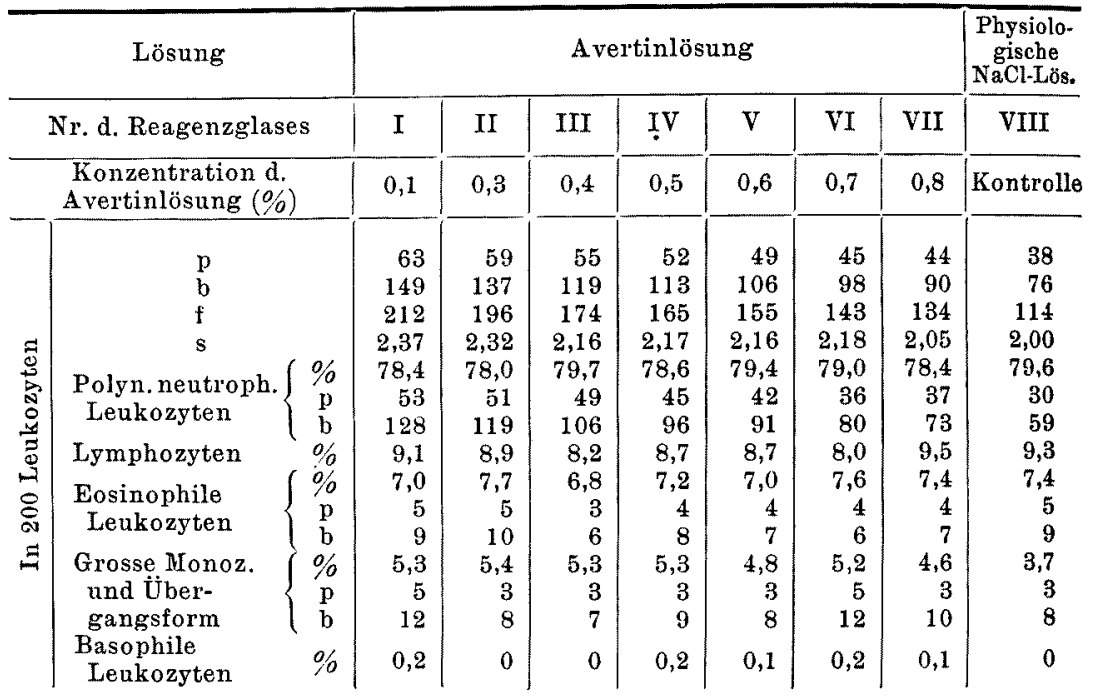

merkt. Unter den Leukozyten nehmen die Neutrophilen die Mehrzahl ein.

\section{Versuch in vitro (2).}

Es wurde am Versuchsresultat in vitro (1) bemerkt, dass das phagozytäre Vermögen jeder Avertinlösung verschiedener Konzentration höher war als die Kontrolle, für welche die Avertin-Konzentration im Blute bei der Narkose als Grundlage bestimmt wurde. Dieser Versuch wurde vorgenommen, um die Frage klarzustellen, in welcher Konzentration die Avertinlösung im Reagenzglas auf die Phagozytose hemmend wirkt.

\section{Versuchsmaterial und -methode.}

Versuchsmaterial: Die Versuchstiere, die Bakterienemulsion, die Leukozytenaufschwemmung und das Serum sind dieselben wie im vorigen $\mathrm{Ab}$ schnitte. Die verwendete Avertinlösung ist diesselbe wie beim Vorversuch. Zur Herstellung verschieden konzentrierten Lösungen stellte ich zunächst bei $40^{\circ} \mathrm{C}$ eine $3,5 \%$ ige, gesättigte Avertinlösung her; die Auflösung war von keiner Zersetzungder Substanz begleitet. Dann fertigte ich die nachfolgenden 7 Avertinlösungen an mit einer um je $0,4 \%$ geringeren Konzentration: 


$\begin{array}{ccc}\begin{array}{c}\text { Nr. des } \\ \text { Reagenzglases }\end{array} & \begin{array}{c}\text { Konzentration der } \\ \text { Avertinlösung für } \\ \text { den Versuch }(\%)\end{array} & \begin{array}{c}\text { Avertin-Konzen- } \\ \text { tration im } \\ \text { Reagenzglas }(\%)\end{array} \\ \text { I } & 1,1 & 0,275 \\ \text { II } & 1,5 & 0,375 \\ \text { III } & 1,9 & 0,475 \\ \text { IV } & 2,3 & 0,575 \\ \text { V } & 2,7 & 0,675 \\ \text { VI } & 3,1 & 0,775 \\ \text { VII } & 3,5 & 0,875\end{array}$

Versuchsmethode ist dieselbe wie im vorigen Abschnitte.

Versuchsresultate.

Tabelle 8.

Versuchsresultat in vitro (2).

(Durehschnittswert an 5 Tieren)

\begin{tabular}{|c|c|c|c|c|c|c|c|c|c|c|}
\hline & Lösung & & & & & tinlö & & & & $\begin{array}{l}\text { Physiolo- } \\
\text { gische }\end{array}$ \\
\hline & Nr. d. Reagenzglas & & I & II & III & IV & $\mathrm{V}$ & VI & VII & VIII \\
\hline & $\begin{array}{l}\text { Konzentration d. } \\
\text { A vertinlösung ( } \%\end{array}$ & & 1,1 & 1,5 & 1,9 & 2,3 & 2,7 & 3,1 & 3,5 & Kontrolle \\
\hline$\Xi$ & $\begin{array}{c}\mathrm{p} \\
\mathrm{b} \\
\mathrm{f} \\
\mathrm{s} \\
\text { Polyn.neutroph. } \\
\text { Leukozyten } \\
\text { Lymphozyten } \\
\text { Eosinophile } \\
\text { Leukozyten } \\
\text { Grosse Monoz. } \\
\text { und Úber- } \\
\text { gangsform } \\
\text { Basophile } \\
\text { Leukozyten }\end{array}$ & $\begin{array}{c}\% \\
\mathrm{p} \\
\mathrm{b} \\
\% \\
\% \\
\mathrm{p} \\
\mathrm{b} \\
\% \\
\mathrm{p} \\
\mathrm{b} \\
\%\end{array}$ & $\begin{array}{r}42 \\
93 \\
135 \\
2,21 \\
87,7 \\
40 \\
90 \\
6,6 \\
3,6 \\
1 \\
1 \\
2,1 \\
1 \\
2 \\
0\end{array}$ & $\begin{array}{r}39 \\
83 \\
122 \\
2,13 \\
87,8 \\
38 \\
82 \\
6,7 \\
3,3 \\
0 \\
0 \\
2,2 \\
1 \\
1 \\
0\end{array}$ & $\begin{array}{r}35 \\
73 \\
108 \\
2,09 \\
92,2 \\
34 \\
72 \\
3,7 \\
2,8 \\
1 \\
1 \\
1,3 \\
0 \\
0 \\
0\end{array}$ & $\begin{array}{r}31 \\
60 \\
91 \\
1,94 \\
93,5 \\
30 \\
59 \\
2,8 \\
2,1 \\
1 \\
1 \\
1,6 \\
0 \\
0 \\
0\end{array}$ & $\begin{array}{r}29 \\
54 \\
83 \\
1,86 \\
93,4 \\
28 \\
53 \\
3,7 \\
1,6 \\
1 \\
1 \\
1,3 \\
0 \\
0 \\
0\end{array}$ & $\begin{array}{r}28 \\
49 \\
77 \\
1,75 \\
94,1 \\
28 \\
49 \\
4,0 \\
0,9 \\
0 \\
0 \\
1,0 \\
0 \\
0 \\
0\end{array}$ & $\begin{array}{r}26 \\
44 \\
70 \\
1,69 \\
93,9 \\
26 \\
44 \\
3,6 \\
1,4 \\
0 \\
0 \\
1,1 \\
0 \\
0 \\
0\end{array}$ & $\begin{array}{r}43 \\
96 \\
139 \\
2,21 \\
88,0 \\
42 \\
95 \\
6,5 \\
3,3 \\
0 \\
0 \\
2,2 \\
1 \\
1 \\
0\end{array}$ \\
\hline
\end{tabular}

Im Durchschnittswert (Tab. 8) bei 1,1\%iger Avertinlösung zeigen $(p),(b),(f)$ und (s) alle nur eine leichte Verminderung im Vergleich zum Kontrollversuch, bei Avertinlösungen mit darüberliegenden Konzentrationen tritt aber mit steigender Konzentration eine zunehmende Verminderung ein, d.h. die Phagozytose der Leukozyten steht bei 1,1\%iger Avertinlösung der des Kontrollversuches etwas nach, aber bei einer 1,5\% igen oder stärkeren Avertinlösung zeigt sich eine offenbare Hemmung. Die benutzte 1,1 und 1,5\%ige Avertin- 
lösung entspricht $0,15125 \mathrm{~g}$, bzw. 0,20625 pro kg Körpergewicht des Meerschweinchens bei intravenöser Narkose. Die erstere Konzentration führt klinisch Atembeschwerden sowie Herzaktionsstörung, zuweilen Atem- sowie Herzstillstand herbei, und die letztere führt immer Atem- sowie Herzstillstand herbei und bewirkt endlich den Tod. Diese Avertin-Konzentrationen sind zweifellos weit grösser als die bei der gewöhnlichen Allgemeinnarkose.

Bei den stärkeren Avertinlösungen ist auch noch Hämolyse zu bemerken.

Der Prozentsatz der Leukozyten ist im grossen und ganzen wie bei der Kontrolle. Die Neutrophilen sind unter den Leukozyten in der Mehrheit.

\section{Zusammenfassung.}

Es wurde von mir beobachtet, dass beim kombinierten Versuch in vivo et in vitro eine grosse Avertinmenge auf die Phagozytose der Leukozyten hemmend, eine kleine dagegen fördernd wirkt, dass aber eine mittlere Avertinmenge (beste Dose der Narkose) von mässigen individuellen Unterschieden abgesehen einerseits hernmend, anderseits fördernd wirkt. Diese Beziehung scheint mit der Arndt-Schulzschen biologischen Regel mehr oder weniger übereinzustimmen.

In Bezug auf den Prozentsatz der Leukozyten beim Kontrollversuch erreichen die Neutrophilen 3 Stunden nach der Narkose den maximalen Wert der Vermehrung, deren Grad im allgemeinen niedrig ist. Dagegen bringt die Avertinnarkose erst nach 6 Stunden unabhängig von der Avertindose und der weiteren Narkosenzeit eine Leukozytose hervor, deren Grad je nach der Avertindose verschieden, jedoch immer weit grösser als beim Kontrollversuch ist. Die Schwankung des Prozentsatzes der Lymphozyten ist gerade entgegengesetzt im Vergleich mit der der Neutrophilen.

Bei den weit stärkeren Avertinlösungen im Reagenzglasversuch —gegenüber der 1,1\%igen Lösung beim Versuch in vitro (2)-, deren Konzentrationen weit grösser als die klinische Dose der Narkose sind und bei denen die Tiere Atembeschwerden, sowie Herzaktionsstörungen, weiter Atem- sowie Herzstillstand erleiden, wird die Phagozytose der Leukozyten gehemmt. Es ist aber infolge der natürlichen Beschaffenheit der Narkotika klar und selbstverständlich, dass bei Lösungen mit so hohen Konzentrationen das phagozytäre Vermögen erniedrigt wird. Es erscheint dabei wichtiger, dass als Aver- 
tinkonzentration die im Blute bei der Narkose vorhandene als Grundlage bestimmt wird. Bei diesen Konzentrationen (Versuch in vitro (1)) wurde die Phagozytose nicht gehemmt, sondern gefördert. Nach den Versuchen in vitro (1) und (2) ist verständlich, dass die Grenze der Avertinkonzentration, welche eine Förderung oder Hemmung der Phagozytose scheidet, sich zwischen $0,8 \%$ und $1,1 \%$ für den Reagenzglasversuch befindet.

Im intraperitonealen Exsudat beim Meerschweinchen, welches 6 Stunden nach der Injektion in Bouillon-Kochsalzlösung aufgesogen wird, nehmen die Neutrophilen den grösseren Anteil der Leukozyten ein, was allgemein der Fall ist. Die Lymphozyten, die grossen Monozyten, sowie die Übergangsformen waren alle in ihrer Zahl gering, und die Leukozytose zeigte überhaupt keine grossen Unterschiede mit der Avertinkonzentration.

Es ist bekannt, dass die Neutrophilen unter den Leukozyten die Phagozytose hauptsächlich leisten. Hier muss man nun die Frage aufwerfen, ob der oben erwähnte Einfluss des Avertins auf die Phagozytose dadurch auftritt, dass es auf die Fresszelle selbst, oder auf die phagozytosefördernde Substanz (Serum oder Blutplasma), oder auf die Bakterien selbst wirkt. Mehrere Beobachtungen über die bisher vorhandenen Narkotika legen diese Fragen nahe. Da ich nun einerseits durch die drei obigen Versuche immer von demselben Meerschweinchen die Leukozyten und das Serum entnahm, um ein Komplizieren der Versuche durch individuelle Unterschiede der Tiere möglichst zu beseitigen und da ich anderseits keine Methode finden konnte, auf dem Versuchswege das Avertin selbst wie ein flüchtiges Narkotikum auszutreiben, so gab ich auf die obigen Fragen bezügliche Versuche auf. Da aber die Leukozy tose immer vorhanden ist, trotzdem der Einfluss des Avertins auf die Phagozytose der Leukozyten je nach der Avertinmenge verschieden ist, so ist leicht zu vermuten, dass der Einfluss meistens darauf beruhen wird, dass das Avertin unmittelbar auf die Neutrophilen selbst wirkt. Die Lösung dieser Frage ist schwierig und wird von künftigen Untersuchungen erhofft.

VII. Schluss.

Nach obigen Versuchsresultaten gelangt man zu dem folgenden Schluss: Wenn auch bei der Avertinnarkose die angewandte Dose des Avertins genügend gross, also dessen Konzentration im Blute genügend stark ist, so lange klinisch die Atembeschwerden, sowie die 
Herzaktionsstörung nicht herbeiführt werden (d. h. bei ruhiger Narkose), wird die Phagozytose der Leukozyten ganz und gar nicht gestört. Wenn aber die Dose weiter vergrössert wird und klinisch die genannten Störungen entstehen, wird die Phagozytose deutlich gehemmt. Dagegen wird sie im Anfangsstadium der Narkose oder kurze Zeit danach gefördert, während die Avertinkonzentration im Blute sehr gering ist.

Daher braucht man den Einfluss des Avertins auf die Phagozytose bei klinischer Narkosendose gar nicht zu berücksichtigen.

Zum Schluss spreche ich meinen hochverehrten Lehrer, Herrn Prof. Dr. Sh. Sekiguchi, für seine liebenswürdige Leitung und Durchsicht dieser Arbeit meinen herzlichsten Dank aus. 